\title{
Smart Parking for Slot Occupancy Detection using Prewitt Edge Detection in OpenCV
}

\author{
Twinkle Singh \\ Computer Science \& Engineering \\ Lakshmi Narian College of Technology \& Science \\ Bhopal, Madhya Pradesh, India
}

\begin{abstract}
In the modern era of advanced parking management system, the automatic parking space detection system has been introduced in the new form. This is an emerging area and attracts computer vision researchers to contribute to this field of technology. Proposed system is based on Prewitt Edge Detection method which is able to recognize the occupied and free space parking slots which may reduces the human efforts. In the field of intelligent vehicles and parking management systems, accuracy is often important because human facilities are required. It is necessary to get accurate results at real-time through which an intelligent parking slot or space can be detected which can be implemented with the newly introduced technique. The system is also capable of recognizing that the vehicle has been partially parked, fully parked or wrong parked. There is no need for human surveillance while parking vehicles in real time. The system is capable of classifying vehicles along with humans as well, through which a right decision can be made on a situation where the human or animal has captured the slots. This is a new era of parking management where computer vision plays an important role.
\end{abstract}

\section{Keywords}

Parking Occupancy Detection, OpenCV, Prewitt, Canny, Edge, Computer Vision.

\section{INTRODUCTION}

The automatic parking slot is the method of locating a special area for detection of occupation that confirms whether it is captured or not. Parking is not systematically managed or it is managed manually. The problem is always to identify free parking spaces. This problem usually occurs in urban areas, where the number of vehicles is more than the availability of parking spaces. Various systems have been developed to provide information about free space for parking, but these systems are either based on hardware sensors or based on nearby View Monitor (AVM). The earlier applicable systems are expensive because they are using more than one camera for each parking slot and using the camera of the fish to identify some empty spaces [1-8]. There are many issues related to the parking area, which are not available to solve any ideal techniques. There is no practically established automated system where parking can be managed automatically with high level accuracy. Drivers must search the slots manually and park their vehicle, which sometimes creates interruptions. The proposed system can solve the issues related to the parking management system. The proposed system is intelligent that the vehicle is partially parked or not. The proposed system also recognizes the speed at real time, which alert the controller about entering or exiting vehicles.

\author{
Safdar Sardar Khan \\ Computer Science \& Engineering \\ Lakshmi Narian College of Technology \& Science \\ Bhopal, Madhya Pradesh, India
}

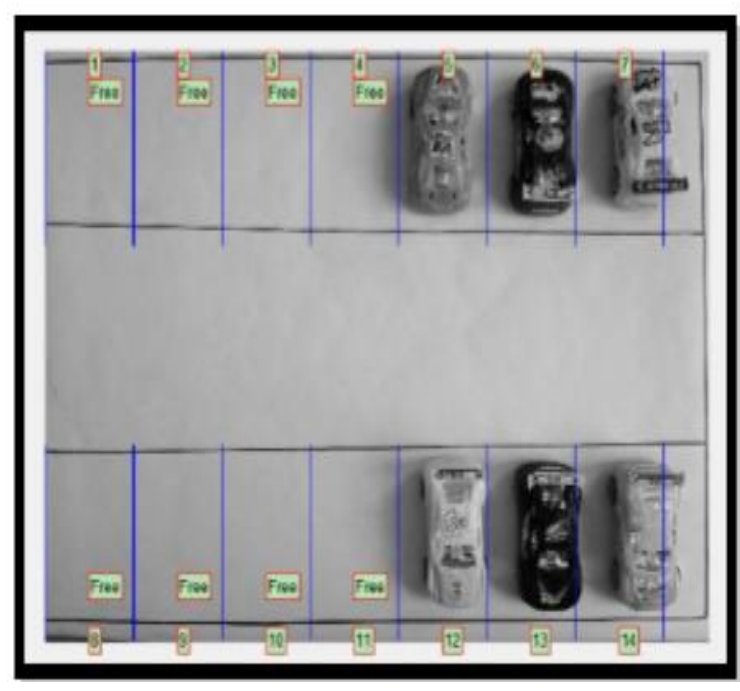

Fig 1: Free Space Detection [1]

\section{RELATED WORKS}

Jae Kyu Suhr et al. proposed a system which is based on Around View Monitor image sequences to recognize the marking of various parking slots. The method which has been used in this paper employs simple detector to find the slots and merges sequentially obtained slots. The system does not display a single slot cluster and ruins the occupation of single slot because the system is waiting for more slots to display the entire cluster. As the hierarchical tree structure approach is used to detect parking slots sequentially using a single AVM image, in a situation where slot junctions are impeded due to adjacent vehicles. Therefore the resulting system shows different missing and incorrectly estimated slots [2].Jae Kyun Suhair et al. again developed a system to develop and track a parking slots with ultra sonic sensor, developed a method recognizes empty slots using ultrasonic sensor which is costly with complicated installations [3].Petar Solic, Ivan Marasovic et al. demonstrated a system using BAP (Battery Assisted Passive) RFID tags which are basically inactive RFID tags with internal batteries and RFID readers. RFID (radio frequency identification) technology is used to detect the existence of the car in the parking slot. In this technique, the BAP tag is modified by changing its battery from the solar cells. BAP tags are centrally positioned on parking grounds of the area. Occupancy slots are detected when the level of light falls below the preset threshold of the BAP tag, due to which the tag information will not be able to broadcast [4]. using almost View Monitor (AVM) system. System 


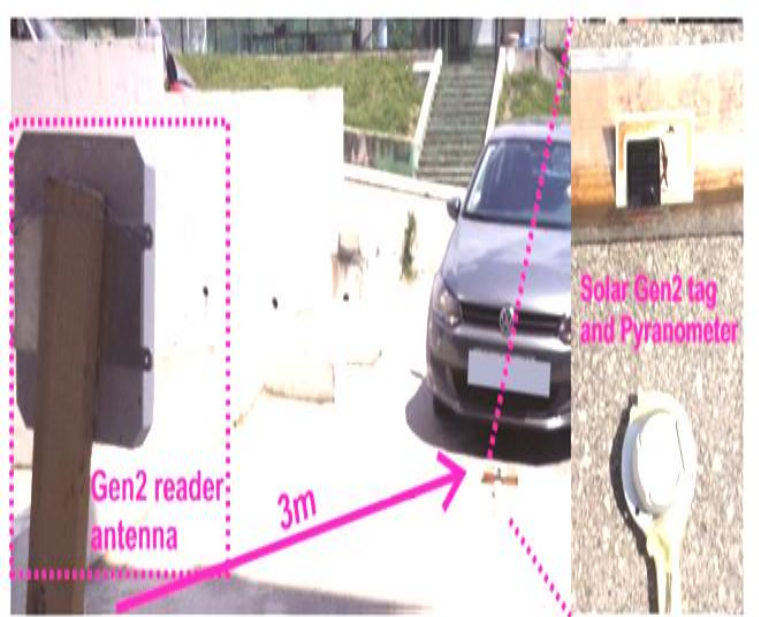

Fig 2: RFID based System [2]

K.Malarvizh et al. proposed a system to detect parking slots using an IOT approach using ultrasonic and proximity sensors. The system is executed in three steps: slot detection, display of parking slots and reservation of empty slots The practical execution of the proposed system in this paper is uncertain for many reasons such as sensors are used such as sensitive to temperature changes, including temperature, dust, water etc. and crowd reservation as a result of mobile reservation facility of parking slots [5].Lei Li, Changle Li et al. developed a method based on Around View Monitor (AVM) system encloses four Fish Eye Cameras around the vehicle. Depending on the extracted information of edges, Line Segment Detector is exploited to identify the marking of parking slots which contains couple of parallel lines with a certain distance in AVM image. Making use of four fish eye camera for every vehicle enhances the cost of whole setup which limits their use at expanded scale as the installed cameras are too expensive in itself [6]. Nazia Bibi et al. proposed a method in which parking assistance system has been developed to recognize the vacant slot and occupied one. Recognition is based on image conversion or correction that allows occupancy detection over a background image. It works like a foreground subtraction method which is highly affected by luminance and obstacles where binary values get changed that resulted incorrect recognition. Hence the system uses traditional approach which is based on density approximation. A small obstacle or pedestrian can affect the precision rate [1].

\section{PROBLEM STATEMENT}

In the field of parking slot detection, the technology developed so far does not provide any accurate approach to practically implementing it. Some systems emphasized the method of hierarchical tree structure using AVM image scenes to observe parking slots and tried to show the cluster of empty slots and avoid showing the sing slot in a cluster, which consumes time. Some techniques have employed ultrasonic sensors that are sensitive to temperature variation and low accuracy. Similarly, RFID is also used in earlier developed techniques which are sensitive to change in nature and can be affected due to increase in dust, water and temperature. The deployment of tags in RFID systems is not adequately trained to detect the presence of a barrier limiting the transmission of information. Base paper proposed a method in which parking assistance system has been developed to recognize the vacant slot and occupied one. Recognition is based on image conversion or correction that allows occupancy detection over a background image. It works like a foreground subtraction method which is highly affected by luminance and obstacles where binary values get changed that resulted incorrect recognition. Hence the system uses traditional approach which is based on density approximation. A small obstacle or pedestrian can affect the precision rate [2-7]. The proposed system may replace the earlier systems which do not possess with intelligent approach.

\section{PROPOSED WORK}

The system proposes real time parking slot occupancy detection using Prewitt edge detection method. Proposed system is able to recognize free space as well as occupied one at real time and also able to inform about the transition of vehicles. No pedestrians or any other obstacles can occupied the parking slots, only vehicles over the parking slots considered as occupied parking. System uses python IDE along with various packages and OpenCV. OpenCV is an Open source Computer Vision library where image processing based operations are stored. Green color is considered as vacant slots and red contouring as occupied one, as shown in the fig 3 .

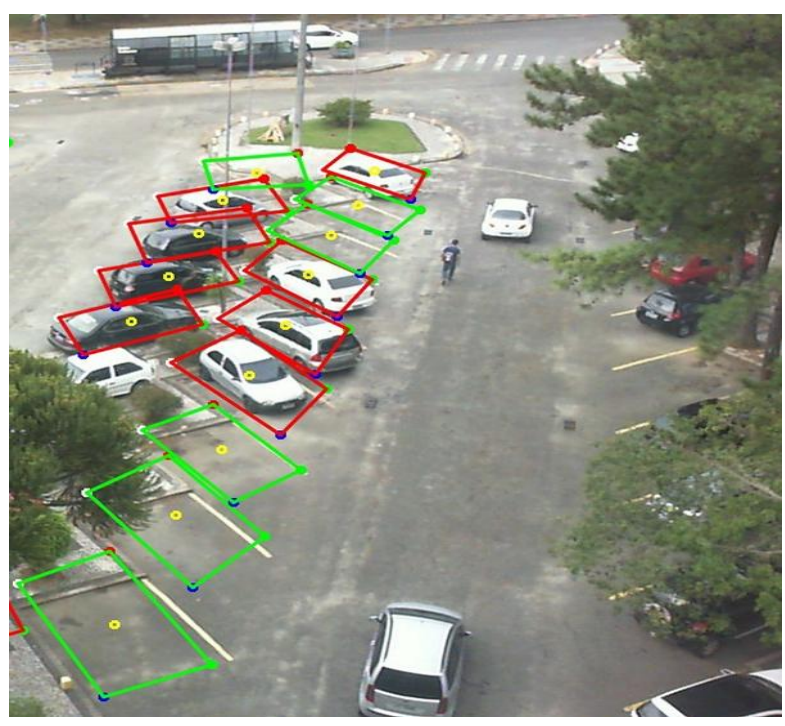

Fig 3: Occupied \& Free Parking Slots

Proposed system can recognize motion at real time for transition alert and classify pedestrian among vehicles. System is classified in various phases such as - Calibration, Input, Tracking, Transition, Classification and Output.

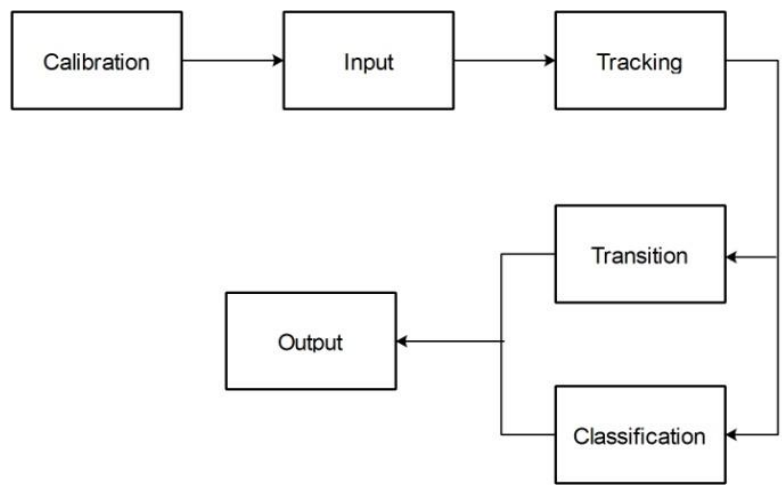

Fig 4: Block Diagram 
Calibration: In calibration, blobs have been assigned as per the dimensions of parking slots with various co-ordinates values. These co-ordinates design sequence of slots along with green and red color for vacant and occupied parking respectively.

Input: Input vehicles as well as pedestrian for further proceeding.

Tracking: After calibration, system will become ready to track vehicles along with pedestrians at real time using Prewitt edge detection.

Transition: The transition process establishes the criteria used to detect when cars enter or leave the parking area. System shows blobs for motion vehicles, it means that system always recognize the real time motion for transition alert when vehicle arrives and depart.

Classification: After recognizing objects over parking areas, it is required to classify whether the object is vehicle or pedestrian for true recognition. Here the classification has been done with aspect ratio of recognized objects because there is a difference between the ratio of pedestrian and vehicles. So, after successfully classifying vehicles and pedestrian, it will notify which parking slot is vacant and which one is occupied along with real time transition alerts.

Proposed system classifying vehicles and pedestrian by identifying through edge using Prewitt edge detection algorithms. Prewitt edge detection is a filter used in image processing or computer vision for detecting edges. Edges classify the aspect ratio of vehicles and pedestrian with high level of accuracy. A quick variation in image brightness of a short spatial distance is known as edge. Here A is considered as input image, $\mathrm{Gx}$ and $\mathrm{Gy}$ are horizontal \& vertical derivative, the computations are as follows:

$$
\begin{aligned}
G_{x} & =\left[\begin{array}{ccc}
+1 & 0 & -1 \\
+2 & 0 & -2 \\
+1 & 0 & -1
\end{array}\right] * A \\
G_{y} & =\left[\begin{array}{ccc}
+1 & +2 & +1 \\
0 & 0 & 0 \\
-1 & -2 & -1
\end{array}\right] * A
\end{aligned}
$$

where $*$ here denotes the 2-dimensional signal processing convolution operation. The $x$-coordinate is defined as increasing in the "left"-direction, and the $y$-coordinate is defined as increasing in the "upward"-direction. At each point in the image, the resulting gradient approximations can be combined to give the gradient magnitude, using:

$$
G=\sqrt{G_{x}^{2}+G_{y}^{2}}
$$

is the gradient magnitude.

$$
\theta=\tan ^{-1}\left(\frac{G_{y}}{G_{x}}\right)
$$

is the gradient direction.

Array shows the directions of edges toward it filtered. Positive value shows the direction of edge detection operation which is required to apply for edge extraction. Before that gradient magnitude and gradient direction have to be computed in order to compute the edges belong to the desired blobs.
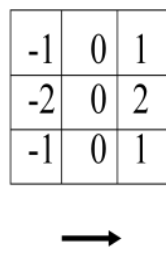

(a)

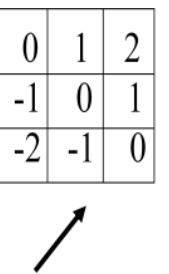

(b)
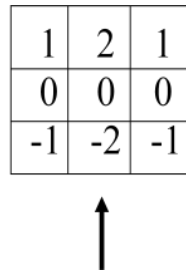

(c)

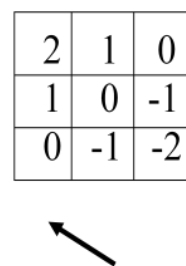

(d)
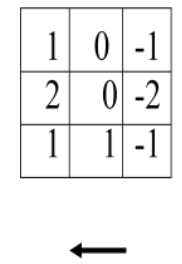

(e)
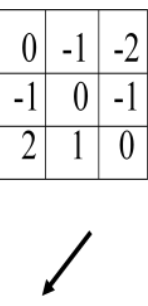

(f)
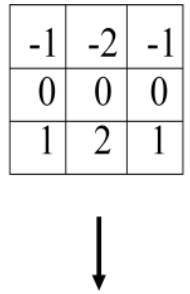

(g)
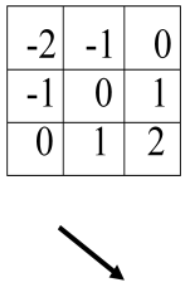

(h)
The arrow shows the direction of edges or gradients. There are eight directions for gradients such as - (a) East, (b) North East, (c) North, (d) North West, (e) West, (f) South West, (g) South, (h) South East, respectively. Let it be more precise with flow chart as shown in fig. 4.1. In flow chart, first of all blobs have been assigned in $\mathrm{x}$ and $\mathrm{y}$ coordinates once the first frame is acquired. Then it will rectify whether frame is about to end or not, if it becomes end or equal to the total number of frames then the process will get end otherwise frame selection will proceed till frame no. will not equal to the last frame. Then Prewitt will be applied for edge extraction that acquires vehicles as well as pedestrian. If edge has been detected inside the blobs (parking slot) then it will confirm whether its aspect ratio is greater than 1 or not, if it is greater than 1 , it means that it is a vehicle otherwise it is considered as pedestrian. It will further examine whether it is greater than the threshold value or not, if it is greater than the threshold value it means that it should be considered as occupied slot otherwise it will be free parking slot or partial one. Threshold value is a value where slot has been occupied with 70 percent of the area and if recognize area is less than the threshold value, it means that vehicles belong to either outside the parking area or partially parked. 


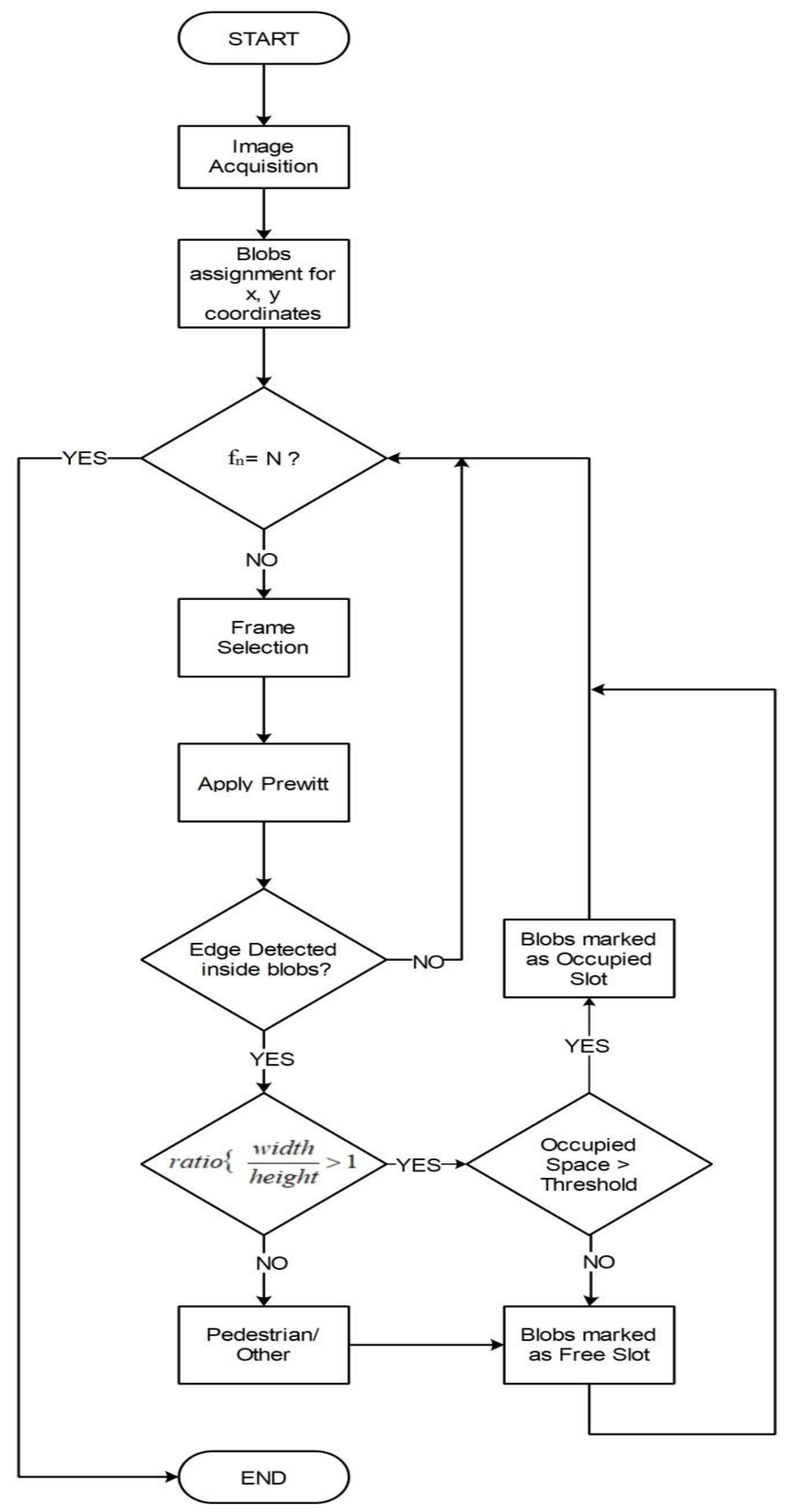

Fig 5: Flow Chart 


\section{PROPOSED METHODOLOGY}

\subsection{Prewitt Algorithm}

Require: Horizontal gradient mask $\mathrm{G}_{\mathrm{x}}$, Vertical gradient mask $G_{y}$, Free slot $P_{s}$, Occupied slot $P_{d}$, Pedestrian P, Vehicle V, threshold value $\mathrm{T}$, Absolute magnitude $\mathrm{G}$, Unknown Object $\mathrm{O}_{\mathrm{b}}$.

Input: A as input image array.

Output: Gradient edges.

Step 1: Acquire the input image.

Step 2: Prewitt gradient function

$$
\mathrm{G}_{\mathrm{x}}=\left[\begin{array}{lll}
+1 & 0 & -1 \\
+2 & 0 & -2 \\
+1 & 0 & -1
\end{array}\right] \quad \mathrm{G}_{\mathrm{y}}=\left[\begin{array}{ccc}
+1 & +2 & +1 \\
0 & 0 & 0 \\
-1 & -2 & -1
\end{array}\right]
$$

Step 3: Apply gradient $\mathrm{G}_{\mathrm{x}}$ and $\mathrm{G}_{\mathrm{y}}$ to input image

$$
\begin{aligned}
& S_{1}=G_{x} * A \\
& S_{2}=G_{y} * A
\end{aligned}
$$

Step 4: Compute the absolute magnitude of the gradient.

$$
|\mathrm{G}|=\sqrt{{S_{1}{ }^{2}+S_{2}{ }^{2}}^{2}}
$$

Step 5: Create blobs for absolute magnitude as V.

Step 6: if ratio (V) $\frac{\text { width }}{\text { heigh }\{>1 \text { then }}$

$\mathrm{O}_{\mathrm{b}}$ considered as vehicle

else

$\mathrm{O}_{\mathrm{b}}$ is considered as pedestrian or other object

\section{end else}

end if

\section{Step 7: if $\mathrm{O}_{\mathrm{b}}>\mathrm{T}$ then}

$P_{s}$ considered as occupied slot $P_{d}$

else

$\mathrm{P}_{\mathrm{s}}$ considered as free slot

end else

end if

Step 8: End

\section{RESULT ANALYSIS}

TP - True Position, TN- True Negative, FP - False Positive, FN - False Negative, A- Accuracy.
Table 1 Result Analysis

\begin{tabular}{|c|c|c|c|c|}
\hline Frames & TP & TN & FP & FN \\
\hline 1338 & 1333 & 5 & 0 & 0 \\
\hline
\end{tabular}

$$
\begin{gathered}
A=\frac{\text { Total no.offrames }-(T N+F P)}{\text { Total } \text { no.offrames }} * 100 \% \\
A=\frac{1338-(5+0)}{1338} * 100 \% \\
A=99.62 \%
\end{gathered}
$$

Table 1 shows the number of vehicles transitions along with their precisions whether it is true negative, true positive, false negative and false positive. Here, true positive means there is vehicle transition occurs inside the parking slots and it is correctly recognized as occupied blobs. True negative means

\begin{tabular}{|c|c|c|}
\hline & Nazia Biwi [1] & Proposed \\
\hline $\begin{array}{l}\text { Total no. of Frames Tested } \\
(\mathrm{N})\end{array}$ & 200 & 1338 \\
\hline $\begin{array}{l}\text { Total no. of Correct } \\
\text { Recognition (FP) }\end{array}$ & 193 & 1333 \\
\hline $\begin{array}{l}\text { Total no. of Incorrect } \\
\text { Recognition (FP) }\end{array}$ & 7 & 5 \\
\hline Overall Accuracy & $98 \%$ & $99.62 \%$ \\
\hline
\end{tabular}
there is transition occurs inside the blob but system shows occupied space as free slot. False positive and false negative is inversely proportional to each other, it means that vehicle has not been transit, but system captured occupied slots and free one respectively.

Table 2 Result Comparison

Table 2 shows the result comparison with previous result where 200 tests have been performed and correct recognition has been calculated as 193 and 7 as incorrect recognition. So, the overall accuracy has been recorded as $98 \%$. In proposed system, 1338 frames have been tested that resulted 1333 as correct recognition and 5 as incorrect recognition and the overall accuracy has been calculated as $99.62 \%$ which is bit higher than the previous one.

\section{CONCLUSION \& FUTURE SCOPE}

The systems which have been proposed till now are costly because most of the systems are using sensors like ultra sonic, proximity and fish cameras which increase the cost of the system since these components needs to install in every vehicle to execute the system. The proposed system is capable enough to efficiently identify vacant and occupied parking slots by using Prewitt edge detection which enhances the accuracy and proficiency of the system up to a great extent. Accuracy is often important for implementing it on real time and a good technique can explore it effectively. In future, a system can be developed with minimal incorrect recognition in aspect to correct recognition. 


\section{REFERENCES}

[1] Nazia Bibi , Muhammad Nadeem Majid , "Automatic Parking Space Detection System", 2nd International Conference on Multimedia and Image Processing, 2017.

[2] Jae Kyu Suhr and Ho Gi Jung "Fully-automatic Recognition of Various Parking Slot Markings in Around View Monitor (AVM) Image Sequences",15th International IEEE Conference on Intelligent Transportation Systems Anchorage, Alaska, USA, September 16-19, 2012.

[3] Jae Kyu Suhr and Ho Gi Jung "Sensor Fusion-Based Vacant Parking Slot Detection and Tracking", IEEE Transactions On Intelligent Transportation Systems, 2013.

[4] Petar 'Solic', Ivan et al. "RFID-based Efficient Method for Parking Slot Car Detection”, IEEE 2015.

[5] K.Malarvizhi, A.Kayathiri, K.Gowri subadra "Survey paper on vehicle parking slot detection using Internet of
Things", International Conference on Computation of Power, Energy, Information and Communication (ICCPEIC), IEEE 2017.

[6] Lei Li1, Changle Li, "Automatic Parking Slot Detection Based on Around View Monitor (AVM) Systems", IEEE 2017.

[7] Jae Kyu Suhr and Ho Gi Jung “Automatic Parking Space Detection and Tracking for Underground and Indoor Environments", IEEE Transactions On Industrial Electronics, 2016.

[8] Sarthak Mendiratta, Debopam Dey and Deepika Rani Sona, "Automatic Car Parking System with Visual Indicator along with IoT”, IEEE, 2017.

[9] Jian-Yu Chen, "A visual method for the detection of Available Parking Slots", IEEE International Conference on Systems, Man, and Cybernetics (SMC) Banff Center, Banff, Canada, October 5-8, 2017. 